\title{
Review of: "A new substrate triggers susceptibility by uncoupling a bacterial multidrug resistance efflux pump"
}

\author{
Graeme Conn ${ }^{1}$, Logan G. Kavanaugh ${ }^{1}$ \\ 1 Emory University
}

Potential competing interests: The author(s) declared that no potential competing interests exist.

Small multidrug resistance (SMR) transporters are common in bacterial species and can efflux toxic compounds to confer resistance phenotypes. The E. coli SMR transporter EmrE transporter was originally defined by its ability to perform coupled 2 proton $(\mathrm{H}+): 1$ drug antiport leading to a drug resistance phenotype. However, more recent studies suggested the potential for EmrE to use variable transport mechanisms, including proton-drug symport, or proton or drug uniport. Uncovering such mechanisms and how they could be promoted would have important implications for bacterial drug resistance and new antimicrobial development.

Beginning from a hypothesis that transporter substrate recognition drives the transport mechanism, the authors used an unbiased screen in E. coli $\triangle e m r E$ complemented with either wild-type EmrE or nonfunctional E14Q-EmrE to identify small molecule substrates that elicit either a resistance (WT EmrE growth favored) or sensitivity phenotype (E14Q-EmrE growth favored). The authors confirmed the viability of this assay by identifying two known substrates of EmrE antiport efflux (resistance), methyl viologen and acriflavine. A third previously unknown resistance substrate (chelerythrine) and three potential "nonantiport" susceptibility substrates, including harmane which was investigated in detail. First, using solid supported membrane electrophysiology (SSME) the sensitivity phenotype in the presence of harmane was shown to be due to uncoupled proton leak. Next, NMR chemical shift perturbations (CSP) were measured in the absence and presence of harmane or the substrate TPP+ for two EmrE variants, the non-functional E14Q-EmrE and S64V-EmrW which possesses unaltered substrate interactions but is less dynamic (producing higher quality NMR spectra). Using this approach the authors determined that TPP+ and harmane both interact with a secondary binding site by NMR chemical shift perturbations (CSP), but only the antiporter substrate TPP+ bound to the higher affinity primary binding site (at E14). Based on these observations, they propose that initial substrate binding to the secondary site unlocks the primary binding site allowing efflux substrates to translocate to this area for antiport. In contrast, harmane remains at the secondary site while still allowing access into the primary site, resulting in uncontrolled proton leak.

Overall, the results deepen our understanding of EmrE function, providing direct evidence_for novel 
transport mechanisms for the well-established SMR efflux transporter. Harmane substrate-induced uncoupled proton leak (uniport) results in decreased cell viability revealing that it may be possible to exploit these switches in transport mode via small molecules allowing for decreased viability via PMF draining. As many other efflux pumps require PMF as a source of function, it is possible that targeting SMR proton leakage would have downstream effects on other multidrug resistant efflux pumps. Additionally, SMR pumps are unique to bacteria making them a bacterial-specific target. The work presented in this manuscript is therefore significant, adds to our understanding of SMR function and appears rigorously performed. However, a number of suggestions to improve the presentation of the work and interpretation of the results are presented below.

\section{Major:}

- To set the scene for this study, more discussion on the compounds effluxed by EmrE and the overall clinical/agricultural relevance of targeting this efflux pump could be presented in the Introduction or Discussion.

- The authors do not provide information on how they acquired their MG1655 this previously described, citation?). Similarly, no information is provided on the complementation plasmid containing WT-emrE or mutant-emrE genes. This includes whether genes of interest were under an inducible promoter.

- The growth (OD600) in Figure $2 \mathrm{f}$ is almost double the other three. Does harmane increase bacterial growth? Or do CC, $\mathrm{MV}^{2+}$, and 18-crown-6-ether influence bacterial growth? WT and E14Q-EmrE growth curves in the absence of the substrates should be shown in the supplementary for comparison. Without them is unknown what "normal" growth is considered to be. Similarly, the strains are described in the Figure 2 legend and text as "grew better" or "grew normally" which is a bit vague - in what way? (Shorter lag time, growth rate in exponential phase, higher density?).

- The authors mention that the "sensitive phenotype" appears around 5-hour mark (Figure 2e,f). What is the phenotypic cut-off for this estimation? Upon observation, it appears the split between phenotypes happens as early has 3 hours in the beginning of log-phase. Does the importance of PMF at this time point remain true?

- There is no mention or supplementary data showing that EmrE levels are comparable in the MG1655 and MG1655 $\mathrm{emrE}:$ :emrE or mutants.

- Putative residues in the secondary binding site are not well defined in the text, although there are specific residues highlighted in the supplementary figures showing NMR spectra/ CSPs. These details 
could be better connected. Have mutations been made in ErmE to test the role of putative residues that might form the secondary binding site? If not, while it would be ideal to perform mutagenesis to test the role of one or more of these residues in harmane interactions, such work might be better suited to a future study (in which case, the authors could consider adding to the Discussion a brief description of how this next step might be tackled)

Minor:

- Colors used in figures are indistinguishable in grey scale

- Inset figure legends would be helpful in Figure 2

- E. coli should be in italics throughout the manuscript

- What are TPP+ and MeTPP+ - neither abbreviation is written out and the reason for using one of the other for different experiments is not explained.

- P2, $3^{\text {rd }}$ para $\mathrm{H}+$ (not superscripted)

- P7 line "The results presented HERE SUGGEST that..."

- P11 (Microarrays) write out LB and Amp; other abbreviations not defined and used once, "NiNTA IMAC" on $\mathrm{P} 13$

- P13 (IC50 assays) "The data was performed ..." should be either "Experiments were performed..." or "Data were acquired..."

- P15 There is a reference to "STAR Methods Key Resources Table" which was not included

- Figure 3 legend: a) remove opening methods sentence; b) needs a description of models in panel a; c) all other panel labels are offset by one $(a=b, b=c$ etc $)$

- Figure 4: in panel $f$, to highlight the absence of the drug/primary site interaction: consider adding a third schematic, equivalent to that in panel e but with the double arrow $x^{\prime} d$ out and perhaps with transparency on the new image to highlight that the state is not accessed.

- Ext Data Fig2 - one compound used at much higher concentration (and not in 2-fold increases like the others); what is the rationale for the concentration choices? Legend should state the meaning of red and black curves.

- Ext Data Fig3 - H+ (non-superscript), +1 drug (elsewhere "drug+"), two sentences ("In the case of..." and "Under our experimental...") have grammatical issues (likely missing word).

- Ext Data Fig4 - No cartoon scheme is shown (legend panel A). All other panel labels offset by one $(B=$ $A$, etc).

- Ext Data Fig 5 - "spectra WERE acquired", state that A and B (shown after residue labels on images) refer to chains A and B. Many abbreviations that may not be defined elsewhere? 